\title{
Modeling and Simulation for Homeland Security
}

\author{
$\underline{\text { K.L. Stamber }}^{\text {a }}$, T.J. Brown ${ }^{a}$, D.J. Pless ${ }^{a}$, and A. Berscheid ${ }^{b}$ \\ ${ }^{a}$ Sandia National Laboratories, Albuquerque, New Mexico, United States of America, ${ }^{b}$ Los Alamos National \\ Laboratory, Los Alamos, New Mexico, United States of America, \\ Email: klstamb@sandia.gov
}

\begin{abstract}
Critical infrastructure and its interdependencies play a vital role in our daily functions. Our economies, government, and way of life depend on the sound function of these integrated systems. Analysis of these systems, however, has historically been limited: system owners and operators have limited information outside their domain space (and that legal impediments often limit their ability to better understand the broader system), and very few entities have the desire to better understand these systems as a whole, let alone their interconnections. The National Infrastructure Simulation and Analysis Center (NISAC) is one such entity.
\end{abstract}

NISAC, a program managed by the U.S. Department of Homeland Security (DHS) and comprised of a core partnership of Sandia National Laboratories (SNL) and Los Alamos National Laboratory (LANL), draws on years of modeling experience of various types, focused on infrastructure, population, and the economy, to examine questions pertinent to DHS regarding the function of economy and critical infrastructure of the United States (see Figure 1).

This paper is designed to provide a background on the NISAC program as a whole, including a description of the legislative and funding history behind the program. Additionally, a review of the program's early days, and the modeling and simulation tools developed for other customers that were repurposes and enhanced to meet homeland security mission space is performed. The beginnings of rapid response analysis are discussed; these grew in significance as the result of events such as Hurricane Katrina, and brought about changes both to processes and tool development. These events also led to a change in NISAC's analytic thought process, from an exclusively reactive one to an increasingly proactive one; this change in thought has led to the development of capabilities to address as-yet unseen events that provide US DHS both with benchmark analyses that can be used in the events of a similar event, as well as capabilities to address similar real-world problems. A review of the path ahead for NISAC highlights both the challenges and potential for the program as it moves into its second decade.

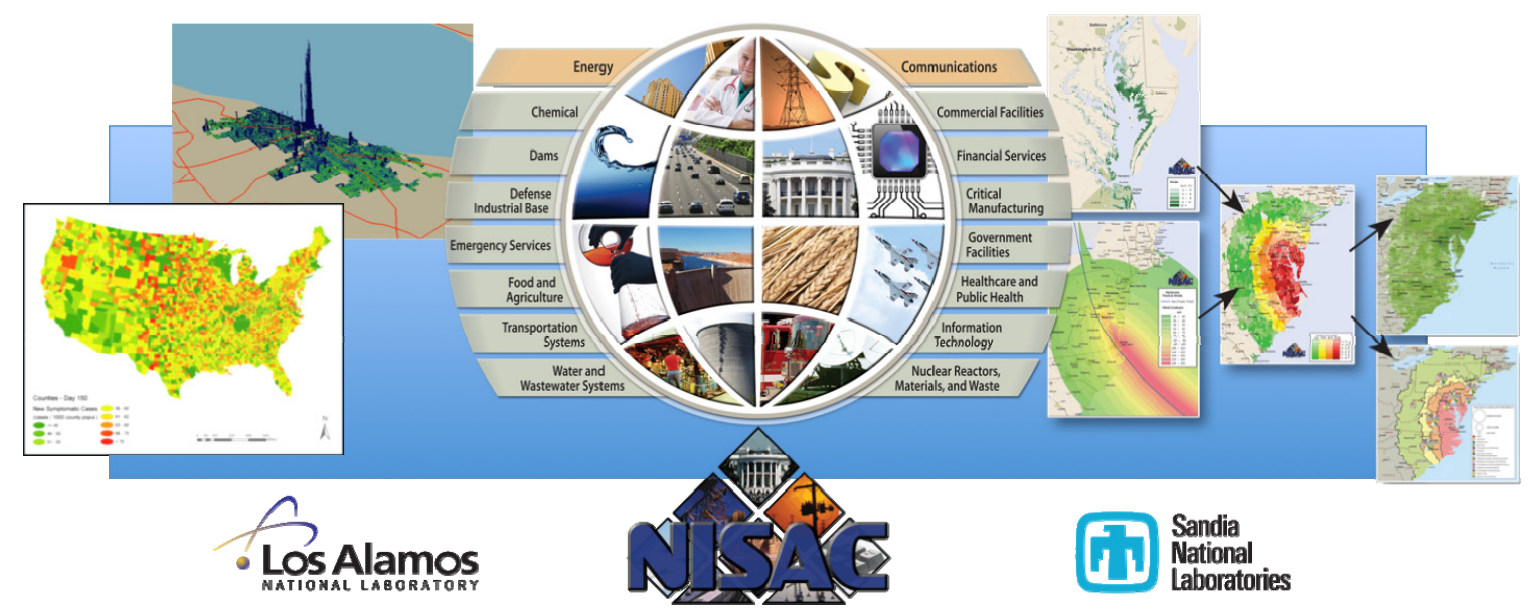

Figure 1. NISAC uses a range of modeling capabilities across the set of critical infrastructure sectors to address questions of consequence to infrastructure, populations, and the economy for hurricanes, earthquakes, pandemics, and other changes to the normal function of systems.

Keywords: critical infrastructure, modeling, analysis, National Infrastructure Simulation and Analysis Center, NISAC 


\section{INTRODUCTION}

For the better part of two decades, United States Government policy has identified the value of critical infrastructure and its interdependencies in maintaining function of the nation's economy, military, government, and private sector, as well as the set of threats - manmade and natural, physical and cyber - that can cause harm to these functions (White House, 1998). Over time, the decomposition of critical infrastructure - and the corresponding responsibility from a federal government perspective - has changed (e.g., U.S. Department of Homeland Security, 2003; White House, 2013). Throughout this period, and in some cases, even preceding it, the Department of Energy National Laboratories - with Sandia National Laboratories (SNL) and Los Alamos National Laboratory (LANL) prominent among them - have conducted work in the development of modeling and simulation capabilities to better understand these sectors, including the performance of these sectors writ large (e.g., Min et al., 2007), causes for failure (e.g., Carrier, 2000), and perspectives on the future state of infrastructure for both planning and risk mitigation (e.g., Nagel et al., 1999; Sandia National Laboratories, 1998). To this end, SNL and LANL began work on the development of the National Infrastructure Simulation and Analysis Center (NISAC), in the late 1990s.

From an initial beginning focused on the development of models and simulations to better understand critical infrastructure, it has developed into a fully mature program, where capability development to help NISAC analysts and U.S. Department of Homeland Security (DHS) customers understand the complex interrelationships among elements of infrastructure and consequences from a range of threats, to consequence analysis using these tools, both in a planned, proactive fashion, as well as in a real-time, reactive fashion, in response to real-world events.

This paper will review the history of NISAC, examine key historical events in context that changed the focus of the program, identify key successes of the last decade and challenges moving forward.

\section{THE NATIONAL INFRASTRUCTURE SIMULATION AND ANALYSIS CENTER (NISAC)}

\subsection{Legislative History}

NISAC is a program managed by DHS and executed by LANL and SNL. Initial funds for NISAC development were provided in October 1999 (Congressional Record, 1999; U.S. House, 1999), and during both this and the following fiscal year, the U.S. Department of Defense (DoD) managed NISAC under the auspices of the Defense Threat Reduction Agency (DTRA).

NISAC was Congressionally established in 2001 (Public Law 107-56, 2001) “...to serve as a source of national competence to address critical infrastructure protection and continuity through support for activities related to counterterrorism, threat assessment, and risk mitigation," and that the support should include "...modeling, simulation, and analysis of the systems comprising critical infrastructures, including cyber infrastructure, telecommunications infrastructure, and physical infrastructure, in order to enhance understanding of the large-scale complexity of such systems and to facilitate modification of such systems to mitigate the threats to such systems and to critical infrastructures generally," and that NISAC should use modeling, simulation, and analysis to both "...provide education and training to policymakers..." as well as "...to provide recommendations to policymakers, and to departments and agencies of the Federal Government and private sector persons and entities upon request, regarding means of enhancing the stability of, and preserving, critical infrastructures."

The U.S. Department of Energy (DOE) Office of Energy Assurance (OEA) managed NISAC for six months at the start of fiscal year 2002. The Homeland Security Act of 2002 (Public Law 107-56, 2002), which established DHS, transferred function and management of NISAC to DHS.

\subsection{Early Capability Development Efforts and Applications}

In its earliest days, NISAC efforts were focused on expanding and refining simulation capabilities leveraged from the laboratories into the NISAC program. Agent and entity-based models of urban traffic congestion (Barrett et al, 1999), and of players in electric power markets (Barton et al., 2000a; Barton et al., 2000b) formed one core of work build on previously funded efforts leveraged by NISAC (e.g., Basu et al., 1998). Additionally, development of system dynamics models was performed on a variety of systems, including telecommunications networks (Jrad et al., 2005; Conrad et al., 2006), seaport operations (Conrad et al, 2003), and integrated models of multiple infrastructure sectors (Brown et al., 2004). Examination of more abstract networks and their application to critical infrastructure systems (Glass et al., 2004) also were brought to bear: These would have future benefits to real-world problems, as shall be detailed below. 
With the transition of NISAC from DoD/DOE to the nascent DHS, development of capabilities to analyze a suite of questions regarding rail transportation and chemicals production, storage and transport (Brown, 2007) helped to show the need for multiple models to address different perspectives of a problem, subject to the questions posed. This fact would grow in importance, as disruptions to critical infrastructure, both real and posited, began to be posed to NISAC by sponsors at DHS.

\subsection{The Beginnings of Fast Analysis}

NISAC began to perform near-real-time analysis of events affecting the nation's critical infrastructure in 2003, with an analysis prior to landfall of Hurricane Isabel, which affected the Washington, DC area. This first test of the ability to use NISAC's modeling capability was limited to a handful of infrastructure sectors energy, telecommunications, transportation, agriculture, ports, and chemicals - and also began a longrunning discussion of the potential impacts to the population and the economy of the affected area posed by such events. Though the only real-time activation for a hurricane in 2003 , it would serve as a structural template that has progressively been modified to address questions of an increasingly larger set of stakeholders. This has come with several changes, as time has progressed:

- Additional infrastructures. Modeling of hurricanes now requires a full examination of all of the critical infrastructure sectors, so as to determine any elements of any sector that might be at risk, and an examination of the effects of those infrastructure systems on other sectors. This is particularly valuable in helping to prioritize restoration of infrastructure services;

- Additional event types. While NISAC's Fast Analysis capability began with earthquakes, it has increasingly been used for additional event types, including other natural disasters (e.g., earthquakes and tsunamis), manmade disasters (e.g., the Deepwater Horizon oil spill), and biological events (e.g., Novel H1N1 Influenza); and

- $\quad$ Reduced turnaround time. The turnaround time on the Hurricane Isabel product to DHS was roughly 60 hours after initial tasking, with a final revision made 84 hours after initial tasking. By comparison, a multi-sector product for Hurricane Isaac in 2012 covering all critical infrastructure sectors as well as population and economic effects, was delivered nine hours after tasking.

These enhancements to NISAC's rapid response are primarily due to the events of the Atlantic hurricane season of 2005, and an identified set of needs, both to develop capabilities to expedite NISAC modeling and analysis, and to develop on-the-shelf analyses that can be used while real-time analysis is planned.

\subsection{The Atlantic Hurricane Season of 2005 and Its Effects}

The Atlantic hurricane season of 2005 featured a significant number of U.S. landfalls, with devastating impact. Through a significant portion of this cycle, DHS tasked NISAC with pre-landfall analysis requests of four storms - Katrina, Rita, Ophelia, and Wilma - which addressed the consequences to infrastructure, population, and the economy of the affected areas resulting from wind damage and storm surge inundation. DHS also tasked NISAC with a number of post-landfall impact analyses for Hurricane Katrina. These analyses were focused on addressing a range of daily concerns, including:

- Revision of pre-landfall economic consequence estimates based on event field data;

- Modeling of consequences to rail transportation and petrochemicals production nationally resulting from data gathered on damaged and disrupted facilities, and on their projected restoration; and

- Identification of elements of critical infrastructure vital to the restoration and recovery of the affected region.

In all, more than 40 separate documents were delivered by NISAC to DHS during this cycle, which included some of the first $24 \times 7$ analytic work performed by NISAC. This sequence, and the analysis performed, also allowed NISAC analysts to understand some of the shortfalls of the existing process, which in turn led to the development of new capabilities.

In terms of modeling consequences of the hurricane, NISAC developed new models to define connectivity among infrastructure assets (Fast Analysis Infrastructure Tool) and to provide resource allocation decision support (LogiSims). NISAC also enhanced and validated a model of power outage likelihood (CICLOPS) and a model of electric power restoration (EPRAM). EPRAM has been continuously validated using a variety of real damage events. A series of post-event validations was completed for the 2004 through 2007 Atlantic hurricane seasons. Documented validations of model output have also been completed for a variety of natural 
disasters such as tornadoes, ice storms, windstorms, and earthquakes. In 2007, an industry audience at the National Hurricane Conference in New Orleans formally evaluated EPRAM model results.

On the geospatial data and mapping front, NISAC analysts built maps "on the fly", often recreating work that had previously been done to capture the same "look and feel" of mapping products included in reports. Following this hurricane season, NISAC standardized a set of maps (with common lookup against networked data sources). This would serve as a starting point for NISAC's FASTMap capability (shown in Figure 2). Today, FASTMap is available to NISAC analysts - and to DHS personnel associated with NISAC - as a web-based mapping service, allowing for publishing-quality maps to be readily produced by a wide range of personnel, all with the same common look and feel.

From an economic modeling perspective, analysis prior to Hurricane Katrina's landfall showed a programmatic weakness, and reliance on a limited

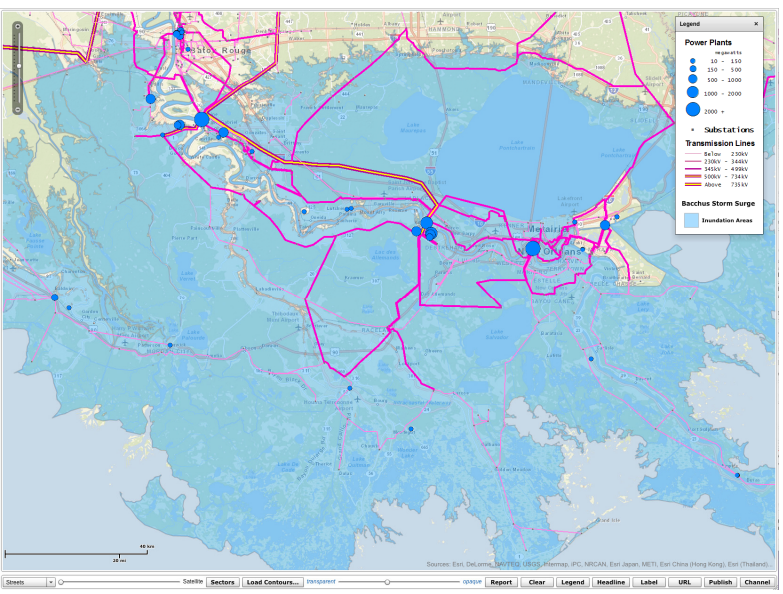

Figure 2. FASTMap allows NISAC analysts to view consequence effects and infrastructure together in a web-based environment, expediting analysis. number of trained economists to produce an Input/Output forecast projection of business interruption effects. In short order following the Atlantic hurricane season, NISAC developed the Regional Economic Accounting (REAcct) tool, which took a geospatial input file (based on power outage and restoration projections developed by NISAC as part of the analytic process) to rapidly calculate the direct and indirect effects associated with an event. Moreover, this method could be readily modified to address other event types. What took 30 economist hours to produce by hand during Katrina can now be completed in minutes, leaving economists time to analyze results of the calculations for DHS to identify points of economic interest and further study.

Next, to meet the demands of both an ever shortening individual product delivery cycle and an extended event delivery cycle, a well-defined process for analysis was scripted between the two labs. This allowed for distinct roles to be performed in the event of NISAC activation, defined information requirements necessary to complete the work to meet deliverables schedules, and was flexible enough to meet changing deliverable requirements (e.g., documents vs. slides).

Finally, to allow time for analysis in the event of a hurricane, NISAC began to develop planned swath analyses outside of hurricane season. These served multiple purposes. First, they created (for select locations with significant population density and risk of hurricane landfall) an on-the-shelf product that could be consulted by DHS in the event of a hurricane taking a track in the direction of one of the analyzed locations. Second, they allowed for testing of new modeling capabilities, data sets, and the like in a controlled, slowmotion environment rather than in the rush of a hurricane event. In total, some 15 of these swaths have been analyzed, and some locations have been re-analyzed to take into consideration new modeling tools, population and economic data, and other changes in process. Additionally, in the last year, this process has taken an additional iterative step of interacting through DHS Protective Security Advisors (PSAs) with local stakeholders. The PSAs have distinct local knowledge and contacts in local government and business communities, allowing for these swath products to be better tailored to the needs and expectations of the local community.

\subsection{Forward-Thinking Modeling and Analysis}

The planned analysis path, which prior to Katrina had seen little traction outside of an occasional task, now became a more central function of NISAC, with the intent to be to analyze the "next Katrina" before it happens. Several productive examples in this space have created additional analyses and capabilities, and allowed for novel questions to be addressed with much less turnaround time than would otherwise be required.

A prime example of the problem spaces NISAC engaged in this area was infectious disease spread. With the World Health Organization reporting occasional cases of a virulent and novel influenza virus, H5N1, NISAC took a different analysis path than other agencies already examining potential outbreak spread. With an already identified set of potential mitigation strategies (including isolation and antiviral treatment of ill 
persons, voluntary household member quarantine and antiviral prophylaxis, social distancing of individuals, school closure, reduction of contacts at work, and prioritized vaccination) NISAC developed outbreak modeling capabilities (Del Valle et al., 2006) and models of the consequences of such an outbreak on socially interacting communities, small (Davey et al, 2008) and large (Stroud et al., 2007), and examined mitigation strategies at a local level (Glass et al., 2005), taking into account the uncertainty of effectiveness of certain measures, such as social distancing. Additionally, NISAC examined the impacts to critical infrastructure operations to see if projected workforce absenteeism associated with an H5N1 outbreak (where prior studies suggested a peak absenteeism of $40 \%$ ) would lead to infrastructure failure. The results of this two-year effort (U.S. Department of Homeland Security, 2007) suggested a multi-layered approach, using social distancing and antivirals to mitigate disease spread.

This work proved fruitful in 2009, as a different influenza, Novel H1N1, appeared in Mexico and the United States. The capabilities used to analyze H5N1 were quickly refined for data received on the new influenza, to provide senior decision makers with revised guidelines for implementation of mitigation strategies.

\subsection{The Path Ahead}

NISAC continues to expand its ability to address questions of national (and international) importance. The path ahead relies on the successful continuance of many existing elements and the proper identification of future problems. First, robustness and accuracy of data continues to be an immediate concern. Licensing requirements for some data sources require their continued funding for utilization. Other data sources, received through Federal agencies, change over time. These changes can lead to disruptions in the ability for models to accurately perform their work, as the data must be in appropriate structures and contain necessary metadata to be used by said models. Efforts such as NISAC Chemical Sector Analysis capability (Ehlen et al, 2010) must be tested periodically (Stamber et al., 2013) to determine model and data validity. Second, continued enhancement of capabilities that expedite the analysis process must be done. Vital to this purpose are those models that have outward-facing interfaces, which enable DHS sponsors to use these capabilities to address immediate questions. This serves as a necessary bulwark for NISAC analysis, enabling the NISAC analyst to get ahead of the current problem. Finally, a continued examination of the problem space is needed, to identify future problems before they occur. NISAC has invested resources in expanding capabilities originally designed for use on domestic problems to incorporate the global perspectives necessary to truly capture the complexity of some infrastructures. This has been particularly true of petroleum fuels and chemicals modeling.

\section{CONCLUSIONS}

From an initial concept, NISAC has expanded into a comprehensive program examining a range of critical infrastructure problems. Over the course of time, as real-world problems have occurred, NISAC has found a space to serve as the analytic arm of the DHS Office of Infrastructure Protection, providing key guidance not only to current problems, but to problems that have yet to occur. Modeling and simulation has been done to keep pace with both a growing problem space and a shrinking rime to respond. Continued model and data validation, capability development to address problems, and examination of unobserved problems are the keys to NISAC continuing to provide useful analysis in the future.

\section{ACKNOWLEDGMENTS}

This research was supported by the National Infrastructure Simulation and Analysis Center (NISAC), a program of the Department of Homeland Security, and comprised of a core partnership of Sandia National Laboratories (SNL) and Los Alamos National Laboratory (LANL). Sandia National Laboratories is a multiprogram laboratory managed and operated by Sandia Corporation, a wholly owned subsidiary of Lockheed Martin Corporation, for the U.S. Department of Energy's National Nuclear Security Administration under contract DE-AC04-94AL85000. Los Alamos National Laboratory is operated by Los Alamos National Security, LLC for the National Nuclear Security Administration of the U.S. Department of Energy under contract DE-AC52-08NA25396. This is document number SAND2013-8485C.

\section{REFERENCES}

Barrett C., R. Beckman, K. Berkbigler, K. Bisset, B. Bush, K. Campbell, S. Eubank, K. Henson, J. Hurford, D. Kubicek, M. Marathe, P. Romero, J. Smith, L. Smith, P. Speckman, P. Stretz, G. Thayer, E. Eeckhout, M.D. Williams (1999). TRANSIMS: Transportation Analysis Simulation System. LA-UR-00-1725. Los Alamos, NM: Los Alamos National Laboratory. 
Barton, D.C., K.L. Stamber (2000a). An Agent-Based Microsimulation of Critical Infrastructure Systems. ENERGEX 2000 - 8th International Energy Forum, Las Vegas, NV, July 2000. SAND2000-0808C, Sandia National Laboratories, Albuquerque, NM.

Barton, D.C., E.D. Eidson, D.A. Schoenwald, K.L. Stamber, R.K. Reinert (2000b). Aspen-EE: An AgentBased Model of Infrastructure Interdependency. SAND2000-2925, Sandia National Laboratories, Albuquerque, NM.

Basu, N., R. Pryor, T. Quint (1998). ASPEN: A Microsimulation Model of the Economy, Journal of Computational Economics 12(3), pp. 223-241.

Brown, T.J., W.E. Beyeler, D. Barton (2004). Assessing Infrastructure Interdependencies: The Challenge of Risk Analysis for Complex Adaptive Systems, International Journal of Critical Infrastructures 1(1), pp. 108-117.

Brown, T.J. (2007). Multiple Modeling Approaches and Insights for Critical Infrastructure Protection. NATO SECURITY THROUGH SCIENCE SERIES D-INFORMATION AND COMMUNICATION SECURITY, 13, 23.

Carrier, P., F. Alvarado, A. Bose, V. Budhraja, W. Buehring, A. Como, C. DeMarco, J. Eto, R. Griego, J. Hauer, I. Hiskens, J. Kueck, T. Overbye, P. Overholt, P. Scalingi, R. Schueler, K. Stamber, R. Thomas, F. C. Zingman (2000). Interim Report of the U.S. Department of Energy's Power Outage Study Team: Findings and recommendations to enhance reliability from the summer of 1999. Department of Energy, March 2000, at http://certs.lbl.gov/pdf/post-final.pdf, accessed 2 July 2013.

Congressional Record (1999). Conference Report on H.R. 2561, 145(109) 8 October 1999, p. H9804.

Conrad, S., W. Beyeler, R. Thomas, G. Hirsch, T. Corbet, T. Brown, G. Hirsch, C. Hatzi (2003). How Do We Increase Port Security Without Imperiling Maritime Commerce? Using Flight Simulators and Workshops to Begin the Discussion, Proceedings of the $21^{\text {st }}$ System Dynamics Conference, July 20-24, 2003, New York, NY, at http://www.systemdynamics.org/conferences/2003/proceed/PAPERS/377.pdf, accessed 2 July 2013.

Conrad, S.H., R.J. LeClaire, G.P. O’Reilly, H. Uzunalioglu (2006). Critical National Infrastructure Reliability Modeling and Analysis, Bell Labs Technical Journal 11(3), 57-71. At http://www.lucent.com/enrich/v1i22007/pdf/BLTJ 20178.pdf, accessed 2 July 2013.

Davey, V.J., R.J. Glass, H.J. Min, W.E. Beyeler, L.M. Glass (2008). Effective, Robust Design of Community Mitigation for Pandemic Influenza: A Systematic Examination of Proposed US Guidance. PLoS ONE 3(7): e2606. doi:10.1371/journal.pone.0002606

Del Valle S.Y., P.D. Stroud, J.P. Smith, S.M. Mniszewski, J.M. Riese, S.J. Sydoriak, D.A. Kubicek (2006). EpiSimS: Epidemic Simulation System. LAUR 06-06714. Los Alamos, NM: Los Alamos National Laboratory.

Ehlen, M.A., P.S. Downes, E.D. Eidson, G.E. Mackey (2010). National Infrastructure Simulation and Analysis Center (NISAC) Value-Chain Modeling of the U.S. Chemical Sector. Proceedings of the American Institute of Chemical Engineers 2010 Spring Meeting \& $6^{\text {th }}$ Global Congress on Process Safety, Tampa, FL.

Glass, R.J., W.E. Beyeler, K.L. Stamber (2004). Advanced Simulation for Analysis of Critical Infrastructure: Abstract Cascades, the Electric Power Grid, and Fedwire. SAND 2004-4239. Sandia National Laboratories, Albuqerque, NM. At http://www.sandia.gov/nisac/wp/wpcontent/uploads/downloads/2012/03/Advanced-Simulation-for-Analysis-of-CriticalInfrastructure.Abstract-Cascades-the-Electric-power-grid-and-Fedwire-2004-4239.pdf, accessed 2 July 2013.

Glass, R.J., L.M. Glass, W.E. Beyeler (2005). Local Mitigation Strategies for Pandemic Influenza. SAMND2005-7955J, Sandia National Laboratories, Albuquerque, NM.

Jones, D.A., C.E. Davis, M.A. Turnquist, L.K. Nozick (2006). Physical Security and Vulnerability Modeling for Infrastructure Facilities, SAND2006-4155, Sandia National Laboratories, Albuquerque, NM.

Jrad, A., H. Uzunalioglu, D.J. Houck, G. O'Reilly, S. Conrad, and W. Beyeler (2005). "Wireless and Wireline Network Interactions in Disaster Scenarios," Military Commun. Conf. (MILCOM'05), Atlantic City, NJ, 2005, pp. 1-7. 
Min, H.J., W. Beyeler, T. Brown, Y.J. Son, and A.T. Jones (2007). Toward Modeling and simulation of critical national infrastructure interdependencies, IIE Transactions, 39(1), 57-71.

Nagel, K., R.J. Beckman, and C.L. Barrett (1999). TRANSIMS for Transportation Planning, LA-UR 984389, Los Alamos, NM: Los Alamos National Laboratory.

Public Law 107-56 (2001). Uniting and Strengthening America by Providing Appropriate Tools Required to Intercept and Obstruct Terrorism (USA PATRIOT ACT) Act of 2001. (115 Stat. 272; Date 26 October 2001; enacted H.R. 3162). At http://www.gpo.gov/fdsys/pkg/PLAW-107publ56/pdf/PLAW107publ56.pdf, accessed 2 July 2013.

Public Law 108-7 (2002), The Homeland Security Act of 2002 (117 Stat. 151; Date 20 February 2003; enacted H.R. 5005). At http://frwebgate.access.gpo.gov/cgibin/getdoc.cgi?dbname=108_cong_bills\&docid=f:hj2enr.txt.pdf, accessed 2 July 2013.

Sandia National Laboratories (1998). US Infrastructure Assurance Strategic Roadmaps: Strategies for Preserving our National Security, SAND98-1496, August 1998.

Stamber, K.L., M.A. Ehlen, M. Pepple, M.E. Welk, H. Deng, C.A. Lewis, L. Clark, J.R. Bryant, D. Jasek (2013). Comparative analysis of the transportation of select petrochemicals, Int. J. Critical Infrastructures, 9(3), pp.226-242.

Stroud P., S. Del Valle, S. Sydoriak. Mniszewski, J. Riese (2007). Spatial Dynamics of Pandemic Influenza in a Massive Artificial Society. Journal of Artificial Societies and Social Simulation 10(4) 9.

U.S. Department of Homeland Security (2003). Homeland Security Presidential Directive 7: Critical Infrastructure Identification, Prioritization, and Protection, at http://www.dhs.gov/homeland-securitypresidential-directive-7, accessed 2 July 2013.

U.S. Department of Homeland Security (2007). National Population, Economic, and Infrastructure Impacts of Pandemic Influenza with Strategic Recommendations.

U.S. House (1999). Making Appropriations for the Department of Defense for the Fiscal Year Ending September 30, 2000, and for Other Purposes (to Accompany H.R. 2561) (106 H. Rpt. 371). At http://www.gpo.gov/fdsys/pkg/CRPT-106hrpt371/pdf/CRPT-106hrpt371.pdf, accessed 2 July 2013.

White House (1998). White Paper: The Clinton Administration's Policy on Critical Infrastructure Protection: Presidential Decision Directive 63, May 22, 1998, at http://www.fas.org/irp/offdocs/paper598.htm, accessed 2 July 2013.

White House (2013). Presidential Policy Directive - Critical Infrastructure Security and Resilience, at http://www.whitehouse.gov/the-press-office/2013/02/12/presidential-policy-directive-criticalinfrastructure-security-and-resil, accessed 2 July 2013. 